\title{
Functional plasma polymers deposited in capacitively and inductively coupled plasmas
}

\author{
Dirk Hegemann, ${ }^{1, a)}$ Enrico Körner, ${ }^{1}$ Shang Chen, ${ }^{2,3}$ Jan Benedikt, ${ }^{2}$ and Achim von Keudell ${ }^{2}$ \\ ${ }^{1}$ Empa, Swiss Federal Laboratories for Materials Science and Technology, Lerchenfeldstrasse 5 , \\ 9014 St. Gallen, Switzerland \\ ${ }^{2}$ Ruhr University Bochum, Research Group Reactive Plasmas, 44780 Bochum, Germany \\ ${ }^{3}$ Nagoya University, Graduate School of Engineering, Nagoya 464-8603, Japan
}

(Received 12 December 2011; accepted 10 January 2012; published online 1 February 2012)

\begin{abstract}
Capacitively and inductively coupled plasmas were investigated in order to deposit functional plasma polymers. Considering plasma chemical and surface processes, comparable films can be obtained with both plasma sources yielding distinctly higher deposition rates for ICP. While the gas phase processes scaled with the energy input into the plasma, the surface processes were controlled by the energy dissipated during film growth (ion bombardment). (C) 2012 American Institute of Physics. [doi:10.1063/1.3681382]
\end{abstract}

Plasma polymerisation is well suited to functionalise material surfaces. Applications such as contact lenses or the wicking of textile fabrics require the permanent incorporation of polar surface groups. ${ }^{1,2}$ For this purpose, oxygencontaining reactive gases are mixed with hydrocarbons in the plasma, in order to obtain cross-linked films with sufficient functional groups. The chemical reaction pathway contains gas phase processes (dissociation by electron impact) and surface processes (association) yielding film growth, but also volatile compounds. ${ }^{3,4}$ These processes as well as ion bombardment at the growing film surface were identified to determine the film properties. ${ }^{5}$ For industrial applications, furthermore, the process speed, i.e., the deposition rate is crucial. Therefore, the deposition of oxygen-functional plasma polymers (a-C:H:O) were compared using capacitively and inductively coupled discharges excited by $13.56 \mathrm{MHz}$ radio frequency (RF). While higher plasma potentials appear in CCP, ICP is distinguished by enhanced electron densities. ${ }^{6,7} \mathrm{~A}$ bulk plasma with well-defined density and temperature can be assumed in both cases. ${ }^{8}$ Ethylene $\left(\mathrm{C}_{2} \mathrm{H}_{4}\right)$ and carbon dioxide $\left(\mathrm{CO}_{2}\right)$ were chosen as process gases performed at a pressure of $10 \mathrm{~Pa}$. For the CCP experiments, a symmetric plasma reactor was used with planeparallel electrodes, $30 \mathrm{~cm}$ in diameter and with a gap of $5 \mathrm{~cm} .{ }^{9}$ The substrates were placed on the RF-driven electrode opposite to the gas inlet. The power input was varied from 10 to $70 \mathrm{~W}$. For the ICP series, an ICP source was mounted inside a recipient facing the sample holder at a distance of $8 \mathrm{~cm}$, where a RF bias of $-10 \mathrm{~V}$ was applied. ${ }^{10}$ The transition into the ICP mode was found to occur at about $80 \mathrm{~W}$ (nominal) power input yielding strongly increasing electron densities, while the power coupling is capacitive at lower values. Thus, the power input was examined in the range from 40 to $300 \mathrm{~W}$. One experiment (at the power of $132 \mathrm{~W}$ ) was also performed with an enhanced bias voltage of $-80 \mathrm{~V}$ at the substrate. Higher ion energies due to the increased sheath voltage $(\sim 90 \mathrm{~V}$ compared to $\sim 20 \mathrm{~V})$ are, thus, obtained during film growth. ${ }^{11}$ Comparable gas flow rates were used in both set-ups.

a)Electronic mail: dirk.hegemann@empa.ch.
The plasma chemical reaction pathway can be controlled by the energy input into the gas phase which is given by the macroscopic reaction parameter, power input per (monomer) gas flow rate, $W / F$. In the microscopic picture, on the other hand, the chemical reactions depend on the electron density $n_{e}$, the rate constants $k$ (depending on electron temperature $T_{e}$ ), the pressure $p$, and the residence time in the active plasma zone $\tau_{a c t}{ }^{5}$ Assuming activation reactions, the deposited mass $R_{m}$ out of the active plasma zone as determined by the flux of film-forming species should follow an Arrheniuslike behavior given by

$$
\frac{R_{m}}{F}=G \exp \left(-\frac{E_{a}}{W / F}\right)
$$

where $G$ is related to the rate of film-forming reactions and $E_{a}$ represents the apparent activation energy (for the predominating activation reactions within the considered regime). This approach can now be used to identify the Arrhenius regime, i.e., the regime where the deposited mass follows Eq. (1).

Furthermore, the densification of the growing film is governed by the energy dissipated during film growth, given by the energy density $\varepsilon$

$$
\varepsilon=\frac{\Gamma_{i} E_{\text {mean }}}{R},
$$

with ion flux $\Gamma_{i}$, mean ion energy $E_{\text {mean }}$, and deposition rate $R$. Based on this approach, the plasma polymerization in CCP and ICP discharges was investigated. ${ }^{5}$ In addition, series with pure $\mathrm{C}_{2} \mathrm{H}_{4}$ discharges were carried out in order to normalize the energy input into the gas phase with respect to film deposition, $W /\left.F\right|_{\text {dep }}$, i.e., the energy deposited in the active plasma zone yielding the flux of film-forming species towards the substrates

$$
\left.\frac{W}{F}\right|_{\text {dep }}=\frac{W}{F} r_{\text {dep }} .
$$

Here, $r_{d e p}=W_{a b s} / W \times d_{a c t} / d_{g a s}$, is the percentage of absorbed power in the discharge multiplied by a geometrical 


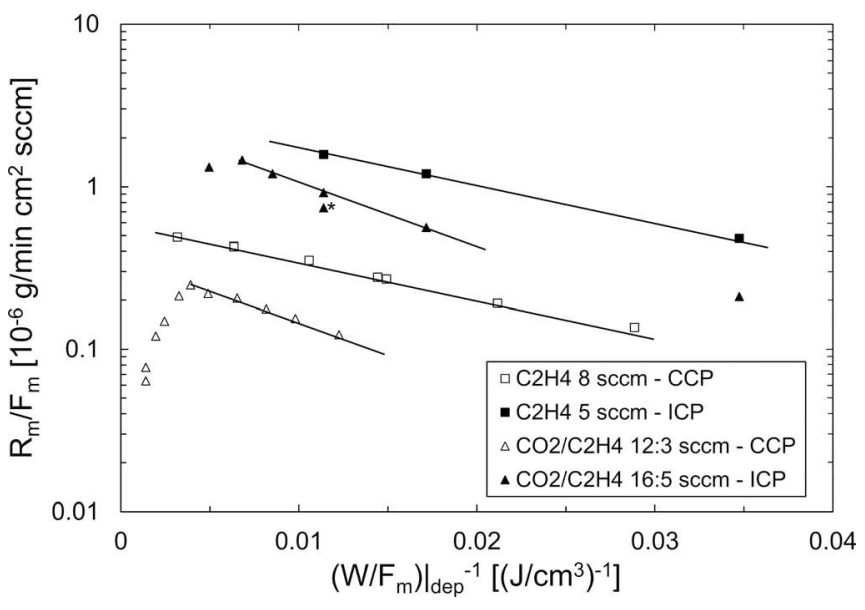

FIG. 1. Arrhenius-type plot of mass deposition rate per monomer gas flow vs. the normalized, inverse energy input for both CCP and ICP discharges. The data point marked with an asterics indicates the usage of an enhanced bias voltage $(-80 \mathrm{~V})$ at the substrate during the ICP discharge.

factor. $^{12}$ The Arrhenius regime was observed over a broad parameter range for both types of plasma sources. Using the well-defined CCP symmetric reactor with $r_{\text {dep }}=0.5$, the activation energy of $\mathrm{C}_{2} \mathrm{H}_{4}$ was found to be $12 \mathrm{eV}$ per molecule. ${ }^{13}$ Here, the vertical gas flow defines $d_{\text {gas }}$ as the electrode gap $(5 \mathrm{~cm})$, while the plasma length yields $d_{a c t}(3 \mathrm{~cm})$. The power absorption was measured to be $80 \%-90 \%$. To obtain a comparable value for ICP, $r_{d e p}=0.05$ has to be taken, which is a factor 10 smaller than the value for the CCP plasma. This is nevertheless reasonable: the power losses are small in the ICP plasma, but the energy is deposited only within a thin skin depth in the ICP plasma $(\sim 1 \mathrm{~cm}){ }^{6}$ In addition, the aspect ratio (electrode distance vs. electrode diameter) is larger compared to the $\mathrm{CCP}$ reactor implying lateral diffusive losses of the reactive species. Both effects apparently lead to a rather small value for the geometrical factor $d_{\text {act }} / d_{\text {gas }}$. Thus, higher nominal power input values are required for the used ICP set-up. Within the Arrhenius regime, a six times higher deposition rate (normalized to the monomer flow rate) was obtained for the ICP discharge (Figure 1).

Next, $\mathrm{CO}_{2}$ was added to the discharge using $12 \mathrm{sccm}$ (with $3 \mathrm{sccm} \mathrm{C}_{2} \mathrm{H}_{4}$ ) for $\mathrm{CCP}$ and $16 \mathrm{sccm}$ (with $5 \mathrm{sccm}$ $\mathrm{C}_{2} \mathrm{H}_{4}$ ) for ICP. The slightly different $\mathrm{CO}_{2} / \mathrm{C}_{2} \mathrm{H}_{4}$ ratios of $4: 1$ and 3.2:1 can still be well compared. ${ }^{14}$ Again, the Arrhenius regime can be identified within the same range of $W /\left.F\right|_{\text {dep }}$, also yielding a comparable slope, i.e., activation energy, for both plasma sources (Figure 1). Compared to pure $\mathrm{C}_{2} \mathrm{H}_{4}$ discharges, the deposition rates are reduced due to the interaction of $\mathrm{CO}_{2}$ (scavenger effects). ${ }^{5}$ For both CCP and ICP discharges, the deposition rate was found to drop at high energy inputs. Since this drop is related to increasing ion bombardment corresponding to an energy density exceeding $1500 \mathrm{eV} \mathrm{nm}^{-3}$ ( $\sim 25 \mathrm{eV}$ per $\mathrm{C}$ atom), the functional plasma polymers were formed at very similar deposition conditions in both discharges considering gas phase (plasma chemical reaction pathway) and surface processes. Comparing the obtained deposition rates (per monomer flow rate), the ICP discharge shows a six times higher rate.

A reduced deposition rate was also obtained when the RF bias at the substrate holder was increased to $-80 \mathrm{~V}$ within the ICP discharge (Figure 1). While this film under-

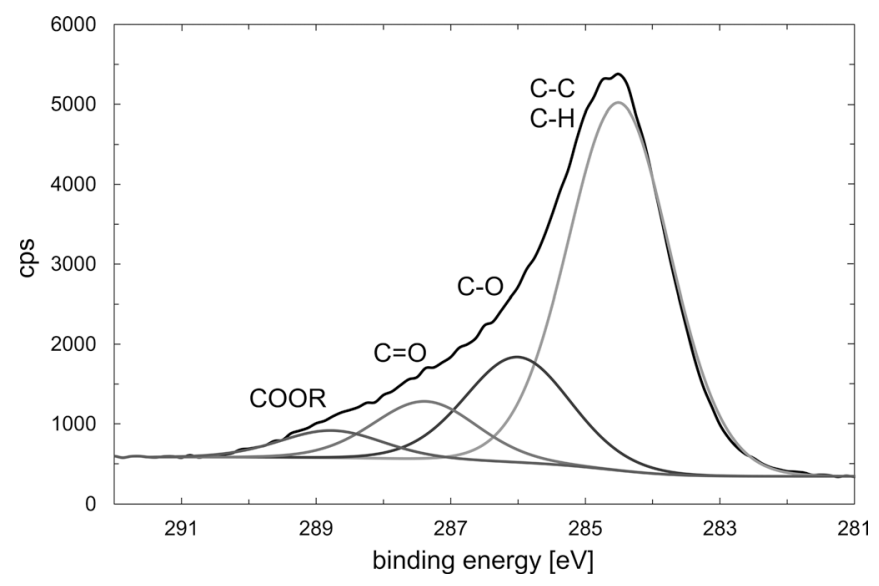

FIG. 2. XPS C1s spectrum of a a-C:H:O plasma polymer deposited with a $\mathrm{CO}_{2} / \mathrm{C}_{2} \mathrm{H}_{4}$ ratio of $4: 1$ using $30 \mathrm{~W}$ of power input (CCP discharge).

went the same plasma chemical reaction pathway (as governed by $W / F)$, stronger ion bombardment due to the higher sheath voltage yielded ion-induced etching and densification.

Within the Arrhenius regime, the $\mathrm{O} / \mathrm{C}$ ratio was found to be nearly constant as measured by XPS and FTIR. ${ }^{15}$ As an example, a typical XPS C1s spectrum is shown in Figure 2. XPS yielded an $\mathrm{O} / \mathrm{C}$ ratio of $0.32( \pm 0.02)$, while a $\mathrm{C}=\mathrm{O}$ band (at $1720 \mathrm{~cm}^{-1}$ ) to $\mathrm{CH}$ band (at $2970 \mathrm{~cm}^{-1}$ ) ratio of 2.9 $( \pm 0.4)$ and a $\mathrm{C}-\mathrm{O}$ band (at $1380 \mathrm{~cm}^{-1}$ ) to $\mathrm{CH}$ band (at $2970 \mathrm{~cm}^{-1}$ ) ratio of $0.34( \pm 0.05)$ was obtained by FTIR for the experimental series with $\mathrm{CO}_{2} / \mathrm{C}_{2} \mathrm{H}_{4}$ (within the Arrhenius regime). As can be seen from Figure 3 , the stronger ion bombardment at the strongly biased substrate results in a reduction of the oxygen-functional group incorporation. The film density was found to increase from 1.3 to $1.5 \mathrm{~g} \mathrm{~cm}^{-3}$ with increasing energy input. ${ }^{14}$ Hence, the same plasma chemical reaction pathway can be assumed yielding oxygen-functional plasma polymers, as long as ion bombardment only leads to a slight densification. Higher film densities $\left(>1.5 \mathrm{~g} \mathrm{~cm}^{-3}\right.$, as obtained for the regime with decreasing deposition rates) yield a loss in functionality and reduced $\mathrm{O} / \mathrm{C}$ ratios.

Hence, the observed six times higher deposition rate within the ICP discharge, which acts to reduce the energy density during film growth, is balanced by the enhanced ion

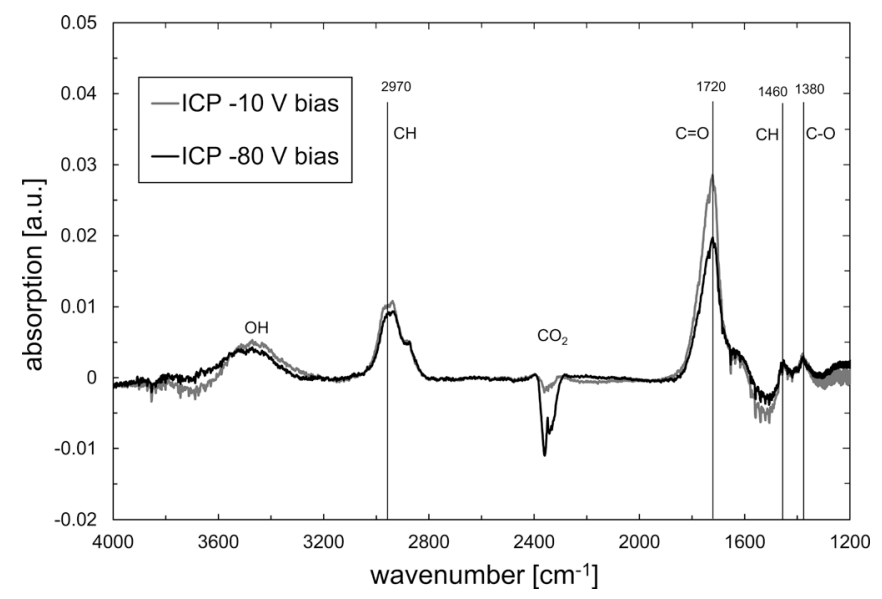

FIG. 3. FTIR absorption spectrum of a a-C:H:O plasma polymer deposited with a $\mathrm{CO}_{2} / \mathrm{C}_{2} \mathrm{H}_{4}$ ratio of $3.2: 1$ using $132 \mathrm{~W}$ of power input (ICP discharge). The film deposited with enhanced RF bias at the substrate shows a reduced $\mathrm{O} / \mathrm{C}$ ratio. 
flux. Both fluxes of film-forming species and ions are roughly proportional to the electron density. The electron densities were found to be one order of magnitude higher in the ICP discharge, while the ion energies might be slightly lower due to the limited sheath voltage. ${ }^{11}$ Therefore, increased electron densities cause an increase in the flux of film-forming species (by a higher number of electroninitiated reactions) as well as an increase in ion flux. The plasma chemical reaction pathway, however, is maintained as determined by the reaction parameter $W / F$ yielding the apparent activation energy $E_{a}$ within the Arrhenius regime. A different electron energy distribution function (different $n_{e}$ and $T_{e}$ ), on the other hand, influences the rate (number per time) of the corresponding reactions, which is only affecting the factor $G$ in Eq. (1), but not the slope. Therefore, the description of plasma polymerization processes on the basis of the microscopic picture is complicated, while the macroscopic approach directly supports process transfer regarding both gas phase and surface processes. Nevertheless, enhanced plasma densities as obtained in ICP discharges can be used to increase the deposition rate of functional plasma polymers.

We can conclude that the same functional plasma polymers can be deposited in both CCP and ICP discharges, as long as the ion bombardment remains moderate avoiding loss of functionality. Nevertheless, cross-linked, stable a-C:H:O coatings were obtained at high deposition rates by optimizing the energy input into the gas phase as well as the energy density during film growth. For ICP, an additional high bias voltage should be avoided in order to retain the functional groups. These findings, thus, imply important principles for applications such as, e.g., in permanent surface hydrophilization (textiles, blood filtration, contact lenses) as well as attachment of chemical and biological molecules (fiber-reinforced composites, drug release, tissue engineering).

${ }^{1}$ H. Yasuda and Y. Matsuzawa, Plasma Process. Polym. 2, 507 (2005).

${ }^{2}$ S. Guimond, B. Hanselmann, M. Amberg, and D. Hegemann, Pure Appl. Chem. 82, 1239 (2010).

${ }^{3}$ D. C. Schram, Pure Appl. Chem. 74, 369 (2002).

${ }^{4}$ R. Reuter, D. Ellerweg, A. von Keudell, and J. Benedikt, Appl. Phys. Lett. 98, 111502 (2011).

${ }^{5}$ D. Hegemann, U. Schütz, and E. Körner, Plasma Process. Polym. 8, 689 (2011).

${ }^{6}$ J. Hopwood, Plasma Sources Sci. Technol. 1, 109 (1992).

${ }^{7}$ H. Ahn, L. Alberts, Y. M. Kim, J. Yu, and K. T. Rie, Surf. Coat. Technol. 169-170, 251 (2003).

${ }^{8}$ M. M. Turner, J. Phys. D: Appl. Phys. 42, 194008 (2009).

${ }^{9}$ E. Körner, G. Fortunato, and D. Hegemann, Plasma Process. Polym. 6, 119 (2009).

${ }^{10}$ M. Schulze, A. von Keudell, and P. Awakowicz, Plasma Sources Sci. Technol. 15, 556 (2006).

${ }^{11}$ C. Hayden, D. Gahan, and M. B. Hopkins, Plasma Sources Sci. Technol. 18, 025018 (2009).

${ }^{12}$ D. Hegemann and U. Schütz, Thin Solid Films 491, 96 (2005).

${ }^{13}$ D. Hegemann, M. M. Hossain, E. Körner, and D. J. Balazs, Plasma Process. Polym. 4, 229 (2007).

${ }^{14}$ D. Hegemann, E. Körner, K. Albrecht, U. Schütz, and S. Guimond, Plasma Process. Polym. 7, 889 (2010).

${ }^{15}$ E. Körner, G. Fortunato, and D. Hegemann, Plasma Process. Polym. 6, 119 (2009). 